\title{
Cutaneous Hyperpigmentation and Familial Gastrointestinal Stromal Tumor Associated with Germline KIT Mutation Treated with Imatinib
}

\section{Wei Yuan}

Zhongshan Hospital Fudan University https://orcid.org/0000-0003-1052-9955

\section{Wen Huang}

Zhongshan Hospital Fudan University

\section{Lei Ren}

Zhongshan Hospital Fudan University

\section{Chen Xu}

Zhongshan Hospital Fudan University

\section{Lijuan Luan}

Zhongshan hospital Fudan University

Jie Huang

Zhongshan Hospital Fudan University

\section{Anwei Xue}

Zhongshan Hospital Fudan University

\section{Yong Fang}

Zhongshan Hospital Fudan University

\section{Xiaodong Gao}

Zhongshan Hospital Fudan University

\section{Kuntang Shen}

Zhongshan Hospital Fudan University

Jinghuan Lv

The Affiliated Suzhou hospital of Nanjing Medical University

Yingyong Hou ( $\square 980455691 @ q q . c o m$ )

Zhongshan Hospital, Fudan University

\section{Case report}

Keywords: Familial gastrointestinal stromal tumor, Germline KIT mutation, Cutaneous hyperpigmentation, imatinib therapy

Posted Date: October 21st, 2020 
DOI: https://doi.org/10.21203/rs.3.rs-92093/v1

License: (c) (i) This work is licensed under a Creative Commons Attribution 4.0 International License. Read Full License 


\section{Abstract}

Background: Familial gastrointestinal stromal tumor (GIST) has been identified with multiple GISTs containing the mutations in germline KIT and PDGFRA. There are only 35 kindreds with germline KIT and 6 with PDGFRA mutations have been reported so far. Familial GIST is often characterized by a series of manifestations, such as multiple lesions, hyperpigmentation, mastocytosis, and dysphagia. Only some kindreds have response to imatinib treatment.

Materials and Methods: A 25-year-old Chinese woman went to hospital because of the abdominal pain, and through the computed tomography (CT) scan showed us the multiple tumors in the small intestine. Her father had a history of multifocal GISTs, and referred to the hospital with abdominal pain and tumor recurrences last year. Immuhistochemical analysis of CD117 and DOG-1 were performed on tumor samples from the two patients, while KIT mutational analysis was carried out by direct sequencing on DNA from paraffin-embedded specimens and saliva sample.

Results: Multiple GISTs associated with diffuse interstitial cells of Cajal (ICC) hyperplasia were illustrated in these two patients. These tumors were positive for CD117 and DOG-1. The germline mutation at codon 560 of exon 11 (p.V560G) of the KIT gene were found. The treatment with imatinib resulted in favorable responses in both tumor and cutaneous hyperpigmentation.

Conclusions: It is difficult to make a correct diagnosis of familial GIST at first time for its rarity. This case was finally diagnosed as familial GIST depending on the combination of diffuse ICC hyperplasia, germline KIT mutation, hyperpigmentation and its family history.

\section{Background}

Gastrointestinal stromal tumors (GISTs) are the most common mesenchymal tumors occurring in the GI tract and originate from the interstitial cells of Cajal ${ }^{1}$. With a wide range of clinical behaviors, prognosis of GISTs varies from indolent disorders with low risks to highly malignant diseases that may metastasize and become lethal ${ }^{2}$. It is known that mutations of the KIT gene have been identified in several human tumors, including multiple GISTs ${ }^{3}$. The KIT gene, a proto-oncogene, is of great significance in the development of various cell lines, which includes the interstitial cells of Cajal and melanocytes ${ }^{4}$. Previous researches have demonstrated that the constitutive activation of tyrosine kinase,a main pathogenic event in most GISTs, caused by the mutation within the KIT gene ${ }^{5}$. Although GISTs are mostly sporadic, families with multiple inherited GISTs associated with germline KIT mutation have been found ${ }^{6}$.

A majority of GISTs, including sporadic and familial GISTs, contain gain-of-function mutations of the KIT, which have been identified among blood relatives with multiple occurrences of GIST $^{7}$. Those patients who do not harbor germline gain-of-function KIT mutation will have a mutation of PDGFRA or other gene mutations ${ }^{8}$. Reported kindreds of familial GIST have represented variable phenotypic features 
characterized by early presentation, multiple lesions, involvement of some related family members, cutaneous hyperpigmentation and dysphagia ${ }^{4}$. Because of the rarity, familial GISTs deserve our paying more attention to the results of molecularly targeted therapy with the selective TKI, such as imatinib ${ }^{4}$,

${ }^{9}$.This paper describes a case of familial GIST in two related patients (father-daughter relationship), which consulted to our center, presenting with multiple GSTs and cutaneous hyperpigmentation, as well as our detection of a single-point germline mutation of KIT in this family. We also monitor their response to imatinib therapy on GISTs and hypopigmentation related to this syndrome.

\section{Case Presentation}

\section{Patient}

\section{Patient 1}

A 25-year-old Chinese woman ( $(: 1$, Fig. 1) presented to the hospital with abdominal pain and progressive cutaneous hyperpigmentation that developed a few months after birth (Fig. 1A). Many new dark lentiginous macules, similar to cafe-au-lait macules (CALMs), had subsequently appeared over her face, body, limbs (Fig. 1B), and also showed both of her popliteal fossa and hands (Fig. 1C). Multiple tumors in the small intestine were showed under the scanning of the computed tomography (CT). A partial resection of the small intestine for multiple tumors, measuring from $0.5 \mathrm{~cm}$ to $4.0 \mathrm{~cm}$, was performed and was diagnosed as GIST in November 2019.

\section{Patient 2}

The Patient 1's 50-year-old father ( $(: 1)$ had a history of GIST in the small intestine 16 years ago, as well as the similar cutaneous hyperpigmentation of skin. He had been admitted to the hospital in 2002 for abdominal pain and dark stools, and then accepted the resection of GISTs. The patient didn't receive imatinib therapy and had no postoperative follow-up. In 2019, he was again referred to the hospital for abdominal pain and was observed and got the results of having tumor recurrences. This patient took the second surgery to remove multiple small intestine tumors (with a maximum diameter of $4.0 \mathrm{~cm}$ ), which diagnosed as multiple GISTs in July 2019. Some small tumors located in specific locations were not surgically removed.

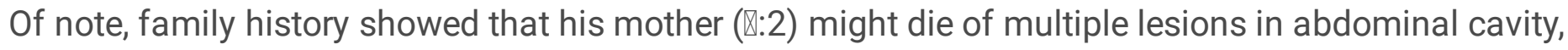
while the diagnosis was not clear at that time. In addition, she was thought to have the same cutaneous

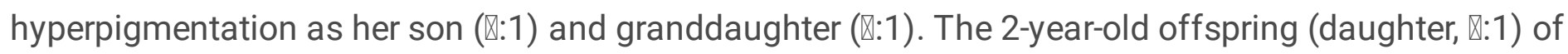
the proband received genetic counseling and review. This child had no manifestations and no cutaneous pigmented lesions. No other family members were affected. For the pedigree of the family see Fig. 2 .

\section{Histopathology and Immunohistochemistry}


The subjects of both cases were re-examination of histopathological and clinical data. Hematoxylin-eosin staining and immunohistochemical analysis of CD117 (the KIT protein product) (A4502; Dako; 1:100), DOG-1 (SP31; CellMarque; 1:100) and Ki67 (Mib1; Dako;1:1000) were performed on $4 \mathrm{~mm}$ formalin fixed paraffin sections of tumor samples (from patient 1 and 2) provided by the first consulting hospital of Suzhou municipal hospital.

\section{Genetic Studies}

After informing consents were obtained from all patients, genomic DNA (gDNA) was prepared from saliva samples and paraffin-embedded specimens of tumor tissues of patient 1 and her father patient 2. Furthermore, we analyzed saliva sample obtained from $\Downarrow: 1$, the daughter of patient 1 , referred to standard methods (Qiagen, Hilden, Germany). Mutation analysis of the KIT and PDGFRA genomic region and flanking sequences (20 bp) were done by polymerase chain reaction (PCR) referred to our previous method $^{10}$.

\section{Results}

\section{Histopathology and Immunohistochemistry}

All of the tumors re-examined were consisted of spindle-shaped cells in a fascicular and whorl-like pattern (Fig. 3), and showed high cellularity, light to medium polymorphic but almost no or a low mitotic rate (patient 1: 0 mitosis/50 HPF, patient 2:0-1 mitosis/50 HPF). A diffuse hyperplasia of the ICCs was observed within the myenteric plexus of the small intestine. The tumor was classified into the group of GIST with low risk for an aggressive clinical behavior of patient 1 (daughter) and high risk of patient 2 (father) respectively.

Immunohistochemistry showed that great majority tumor cells (spindle-shaped cells) were positive for CD117 (KIT receptor) and DOG-1 in both patients, and the Ki67 index were below 1\%.

\section{Mutational Status}

KIT and PDGFRA mutations were analyzed using previously described methods. The results showed a similar point mutation at codon 560 in patient 1 and 2, which resulted in the exchange of leucine by proline (p.V560G), indicating a de novo germline KIT mutation. Furthermore, we analyzed saliva sample that obtained from $\mathbb{1}: 1$ (the daughter of patient 1), and sequence analysis of saliva DNA showed a wildtype sequence in exon 11 of the KIT gene. No additional mutations of KIT exons 9, 13, or 17 as well as PDGFRA exons 12 or 14 were presented in these three family members.

\section{Treatment and Follow-up}

Two months after the tumor resection, patient 1 ( $(: 1)$ was followed up with adjuvant imatinib therapy, $400 \mathrm{mg} / \mathrm{d}$, in case of the recurrence. Three months after treatment initiation, the patient was noticed a decrease in the number of pigmented lesions around her mouth (Fig. 1E), and we can see that the skin on 
her hands turned to light (Fig. 1D), and the color of her hair, pubic hair, and body hair both changed to white. In August 2020, she underwent CT scan, and there has been no clinical evidence of progression.

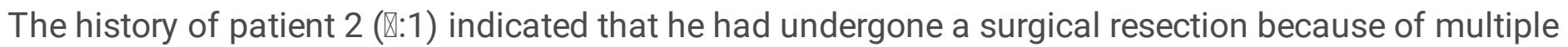
tumors in the small bowel in 2003,and these tumors were histologically diagnosed as GISTs. This patient did not accept imatinib therapy and follow-up after the surgery. The clinical behavior of the tumors appeared relatively indolent. After 16 years (in 2019), recurrence of GIST in the small bowel could be seen. Then the patient took the second surgery and an adjuvant treatment with imatinib $400 \mathrm{mg} / \mathrm{d}$ was carried out. Initiation of imatinib resulted in rapid tumor regression, followed by long-lasting disease stability. Also, hyperpigmentation of the skin diminished within 3 months of imatinib treatment. At his last follow-up visit, CT scan showed a decline of the tumor size and stable disease in other locations.

\section{Discussion}

Familial GIST was first reported by Nishida T in $1998^{11}$. It is a very rare inherited disease and has been reported in 35 different families and 8 individuals with germline KIT mutations so far ${ }^{12}$. Most affected families, including the members described in our study, have been shown to harbor a germline KIT mutation in exon 11 (encoding the juxtamembrane domain) ${ }^{9}$, 13 . While previous studies showed that some GIST families which had been confirmed with a germline PDGFRA mutation ${ }^{1,14}$. In the family described by Nishida et al. ${ }^{15}$ that 1 of 2 consecutive Val residues (codons 559 and 560, GTT-GTT) of the KIT JM structural domain was missing, in which 2 family members showed skin pigmentation in the perineum. By histologic and immunohistochemical examinations, as well as molecular genetic analysis, we uncovered a single germline mutation in codon 560 (c.560T>G, p.V560G) at KIT exon 11 in a family with GISTs and hyperpigmentation.

The origin of mesenchymal tumors in the gastrointestinal tract has long been served a focus of debate. Previous studies ${ }^{16}$ have demonstrated that abnormalities of ICC, the "pacemaker cells of the gut", may cause various motility disorders of the gastrointestinal tract. Miettinen et al. ${ }^{17}$ argued that GISTs were derived from transformed neoplastic precursors of the ICC. ICC hyperplasia and multiple cecal physicians were produced in mice transfected with the knock-in mutant KIT (V558del) ${ }^{18}$. In this study, the diffuse ICC hyperplasia, presented in the muscularis propria in the non-tumorous wall of the small bowel, could be found in two patients. Immunohistochemical examination of tumors resected from patients $₫: 1$ and $\gtrsim: 1$ showed that most tumor cells were positive for DOG-1 and CD117 (KIT protein). What's more, ICC were confirmed to express not only CD117, but also DOG-1. Therefore, data from a transgenic mouse model ${ }^{19}$, together with histologic findings in previous researches ${ }^{20}$ and ours, indicated that activation of germline KIT results in hyperplasia of the ICC cells from which GISTs derive.

Clinically, symptoms such as multiple primary neoplasms (with several affected relatives), dysphagia and abnormal pigmentation 1, 13, 21 can be observed in familial GISTs. Median age at diagnosis of sporadic GISTs is around 60 years, while tumors of familial GISTs may present at earlier ages than that of 
sporadic GISTs ${ }^{21}$. The kindred in our study demonstrated some of these traits. It is worth noting that patient $\otimes: 1$ and $\rrbracket: 1$ (with the same mutation)who were diagnosed with multiple GISTs in their $20 \mathrm{~s}$ and $30 \mathrm{~s}$ were also noted to have cutaneous pigmentation which developed a few months after birth and increased

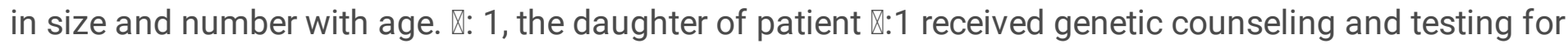
early detection of GIST. Prior researches of familial GISTs have illustrated an association with cutaneous hyperpigmentation ${ }^{22}$. This association results from the role of KIT in development of melanocytes, as well as ICCs from which GISTs derive ${ }^{23}$. One of the dead family member (grandma of $\mathbb{\otimes}: 1$ ) in this study in earlier generation ( $\mathbb{2}: 2)$ indicated that "multiple lesions in abdominal cavity" might be the cause of death. The diagnosis was not clear at that time, probably because GISTs had not yet been considered as a unique clinicopathological entity.

Although the effectiveness of imatinib, a tyrosine kinase inhibitor, in the treatment of familial GISTs had not been defined yet, many individuals with this disease accepted the therapy of imatinib, which might result in resolution of the hyperpigmentation and stable disease ${ }^{3,24}$. However, not all patients experience pigmentary changes and tumor regression in response to imatinib, nor did all patients experience the same degree of changes ${ }^{22}$. Conca et al. ${ }^{25}$ showed GIST in two family members with the L576P mutation (in exon 11) who had a poor response to imatinib since the tumors persisted microscopically. What's more, Divya G et al. ${ }^{9}$ reported two siblings of hereditary GISTs with the missense mutation $\mathrm{p}$. (Val559Ala). Both patients were treated with imatinib, but only one patient's condition changed to stable after such treatment; after a stable period of more than one year, progression of the disease could be observed in another. The different responses to imatinib may due to secondary drug resistance, the type of mutation and its location. Patients in our study were treated with adjuvant imatinib ( $400 \mathrm{mg} / \mathrm{day}$ ) after surgery ( $\mathbb{1}: 1$ after second surgery), within several months of imatinib treatment these hyperpigmentation diminished and the skin tone became lighter. Previous studies have demonstrated that KIT and its ligand stem cell factor (SCF) are considered to play significant roles in the development of 4 cell lineages: hematopoietic cells, melanocytes, germ cells, and mast cells ${ }^{24,26}$. As it is known to all, imatinib has been identified as the inhibition of KIT, which may leads to downstream inhibition of tyrosinase gene promoter and subsequent melanin synthesis inhibition. In vitro studies ${ }^{22,27}$, researchers have reported that melanocytes with decreased proliferation in fibroblasts and low tyrosinase activity can be observed in imatinib-treated skin. This indicates that SCF-KIT interaction can regulate the development and survival of melanocytes in the context of GISTs ${ }^{28}$.

Although GIST associated with germline KIT mutations is very rare, it should be taken into consideration when we encounter patients with multiple lesions, cutaneous hyperpigmentation, multiple lentigines, mastocytosis and other typical manifestation. We should emphasize the importance of inquiring about a detailed family history in individuals with abnormal pigmentation, especially any history of GI lesions. What's more, the possibility of genetic testing and counseling to distinguish individuals with high risk from those with little/no risk is essential. Detecting for the KIT mutation should prior to the start of imatinib therapy, for it can help predicting tumor response. Imatinib as a therapeutic inhibition in these rare disease remains to be determined, mainly because data on therapeutic effects of imatinib in familial 
GISTs is limited. In addition, careful monitoring for the development of GIST (during and after treatment) is reasonable. The specific secondary mutations can direct the clinician to select other appropriate second-line treatment of patients with imatinib resistance.

\section{Abbreviations}

GIST gastrointestinal stromal tumor

CT computed tomography

ICC interstitial cells of Cajal

CALMs cafe-au-lait macules

gDNA genomic DNA

PCR polymerase chain reaction

\section{Declarations}

\section{Ethics approval and consent to participate}

The use of human specimens from patients in the present study was approved by the Ethics Committee of the Zhongshan Hospital Fudan University. All experiments were performed in accordance with the ethical standards of the 1995 Declaration of Helsinki, as revised in Tokyo in 2004.

\section{Consent for publication}

Written informed consent was obtained from all patients.

\section{Availability of data and materials}

All data generated or analyzed during this study are included in this published article

\section{Competing interests}

The authors declare that they have no competing interests.

\section{Funding}


This work was financially supported by Shanghai 305 Municipal Commission of Science and Technology (No. 19441904000), Shanghai Municipal Key 306 Clinical Specialty (No. shslczdzk01302).

\section{Authors' contributions}

WY and WH conducted the studies, and are the primary Authors of this article. WY performed the related experiments, and WH drafted the article. RL, CX, Lj L, JH collected specimens and the history data. Aw X, YF, Xd G, Kt S developed treatment plans for the patients and follow-up. Jh $L$ summarized the clinical and histologic data. Yy $\mathrm{H}$ conceived the project, revised the article, obtained the funding, and designed the research. All Authors met the authorship criteria and read and approved the final article.

\section{Acknowledgements}

The Authors sincerely express their thanks for the financial support of the funds for our research (see under Funding) and grate fully acknowledge our colleagues and many individuals for their help during the study processes.

\section{References}

1. Li FP, Fletcher JA, Heinrich MC, et al. Familial gastrointestinal stromal tumor syndrome: phenotypic and molecular features in a kindred. J Clin Oncol. 2005;23:2735-2743.

2. Dematteo RP, Heinrich MC, El-Rifai WM, Demetri G. Clinical management of gastrointestinal stromal tumors: before and after STI-571. Hum Pathol. 2002;33:466-477.

3. Wali GN, Halliday D, Dua J, leremia E, McPherson T, Matin RN. Cutaneous hyperpigmentation and familial gastrointestinal stromal tumour associated with KIT mutation. Clin Exp Dermatol. 2019;44:418-421.

4. Carballo M, Roig I, Aguilar F, et al. Novel c-KIT germline mutation in a family with gastrointestinal stromal tumors and cutaneous hyperpig mentation. Am J Med Genet A. 2005;132a:361-364.

5. Hirota S, Isozaki K, Moriyama Y, et al. Gain-of-function mutations of c-kit in human gastrointestinal stromal tumors. Science. 1998;279:577-580.

6. Sekido Y, Ohigashi S, Takahashi T, Hayashi N, Suzuki K, Hirota S. Familial Gastrointestinal Stromal Tumor with Germline KIT Mutations Accompanying Hereditary Breast an d Ovarian Cancer Syndrome. Anticancer Res. 2017;37:1425-1431.

7. O'Riain C, Corless CL, Heinrich MC, et al. Gastrointestinal stromal tumors: insights from a new familial GIST kindred with unusual genetic and p athologic features. Am J Surg Pathol. 2005;29:1680-1683.

8. Robson ME, Glogowski E, Sommer G, et al. Pleomorphic characteristics of a germ-line KIT mutation in a large kindred with gastrointestinal stro mal tumors, hyperpigmentation, and dysphagia. Clin 
Cancer Res. 2004;10:1250-1254.

9. Gupta D, Chandrashekar L, Larizza L, et al. Familial gastrointestinal stromal tumors, lentigines, and caf "' |-au-lait macules associated with germli ne c-kit mutation treated with imatinib. Int J Dermatol. 2017;56:195-201.

10. Hou YY, Grabellus F, Weber F, et al. Impact of KIT and PDGFRA gene mutations on prognosis of patients with gastrointestinal stromal tumors after complete primary tumor resection. J Gastrointest Surg. 2009;13:1583-1592.

11. Nishida T, Hirota $S$, Taniguchi $M$, et al. Familial gastrointestinal stromal tumours with germline mutation of the KIT gene. Nature Genetics. 1998;19:323-324.

12. Hasegawa M, Shimizu A, leta K, et al. Generalized lentigines associated with familial gastrointestinal stromal tumors dramatically improved by imatinib treatment. J Dermatol. 2020;47:e241-e242.

13. Jones DH, Caracciolo JT, Hodul PJ, Strosberg JR, Coppola D, Bui MM. Familial gastrointestinal stromal tumor syndrome: report of 2 cases with KIT exon 11 mutation. Cancer Control. 2015;22:102108.

14. Chompret A, Kannengiesser $C$, Barrois $M$, et al. PDGFRA germline mutation in a family with multiple cases of gastrointestinal stromal tumor. Gastroenterology. 2004;126:318-321.

15. Zsebo KM, Williams DA, Geissler EN, et al. Stem cell factor is encoded at the SI locus of the mouse and is the ligand for the c-kit tyrosine kin ase receptor. Cell. 1990;63:213-224.

16. Hirota S, Okazaki T, Kitamura Y, O'Brien P, Kapusta L, Dardick I. Cause of familial and multiple gastrointestinal autonomic nerve tumors with hyperplasia of interstiti al cells of Cajal is germline mutation of the c-kit gene. Am J Surg Pathol. 2000;24:326-327.

17. Miettinen M, Lasota J. Gastrointestinal stromal tumors-definition, clinical, histological, immunohistochemical, and molecul ar genetic features and differential diagnosis. Virchows Arch. 2001;438:1-12.

18. Gromova P, Ralea S, Lefort A, et al. Kit K641E oncogene up-regulates Sprouty homolog 4 and trophoblast glycoprotein in interstitial cells of Cajal in a murine model of gastrointestinal stromal tumours. J Cell Mol Med. 2009;13:1536-1548.

19. Maeyama $\mathrm{H}$, Hidaka $\mathrm{E}, \mathrm{Ota} \mathrm{H}$, et al. Familial gastrointestinal stromal tumor with hyperpigmentation: association with a germline mutation of the c-kit gene. Gastroenterology. 2001;120:210-215.

20. Moskaluk CA, Tian Q, Marshall CR, Rumpel CA, Franquemont DW, Frierson HF. Mutations of c-kit JM domain are found in a minority of human gastrointestinal stromal tumors. Oncogene. 1999;18:18971902.

21. Kuroda N, Tanida N, Hirota S, et al. Familial gastrointestinal stromal tumor with germ line mutation of the juxtamembrane domain of the KI T gene observed in relatively young women. Ann Diagn Pathol. 2011;15:358-361.

22. Coleman E, Panse G, Cowper S, Lacy J, Leventhal J. Disappearing pigmentary mosaicism during imatinib treatment for gastrointestinal stromal tumors. JAAD Case Rep. 2019;5:170-172. 
23. Shibusawa Y, Tamura A, Mochiki E, Kamisaka K, Kimura H, Ishikawa O. c-kit Mutation in generalized lentigines associated with gastrointestinal stromal tumor. Dermatology. 2004;208:217-220.

24. Farag S, van der Kolk LE, van Boven $\mathrm{HH}$, et al. Remarkable effects of imatinib in a family with young onset gastrointestinal stromal tumors and cutan eous hyperpigmentation associated with a germline KIT-Trp557Arg mutation: case report and literature overview. Fam Cancer. 2018;17:247-253.

25. Conca E, Negri T, Gronchi A, et al. Activate and resist: L576P-KIT in GIST. Mol Cancer Ther. 2009;8:2491-2495.

26. Antonescu CR. Gastrointestinal stromal tumor (GIST) pathogenesis, familial GIST, and animal models. Semin Diagn Pathol. 2006;23:63-69.

27. Cario-Andr“|' M, Ardilouze L, Pain C, Gauthier Y, Mahon FX, Taieb A. Imatinib mesilate inhibits melanogenesis in vitro. Br J Dermatol. 2006;155:493-494.

28. Leong KW, Lee TC, Goh AS. Imatinib mesylate causes hypopigmentation in the skin. Cancer. 2004;100:2486-2487; author reply 2487-2488.

\section{Figures}
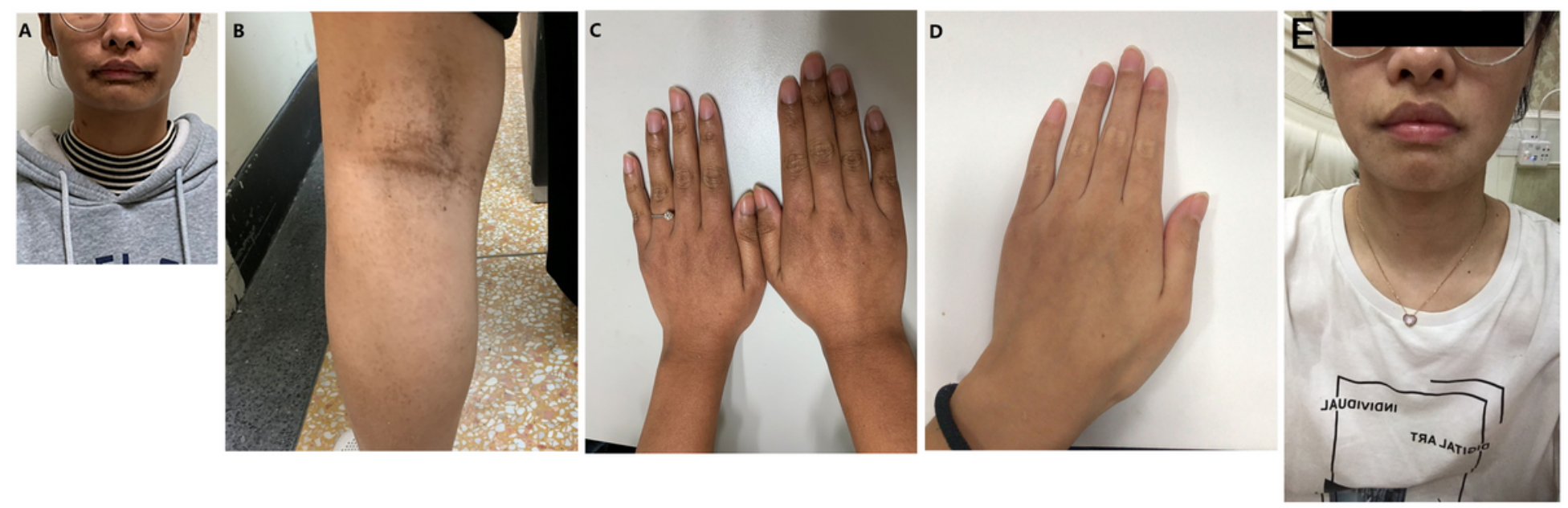

\section{Figure 1}

Clinical features of pigmented lesions of patient $1(\mathbb{\otimes}: 1)$. Hyperpigmentation with well-defined margin were observed on the (A) face and around the mouth (B) back of the lower limbs. (C) Before initiation of imatinib therapy there was apparent pigmentation on the hand. (D) (E) After seven months of imatinib therapy the pigmentations diminished, and the skin tone became lighter. 


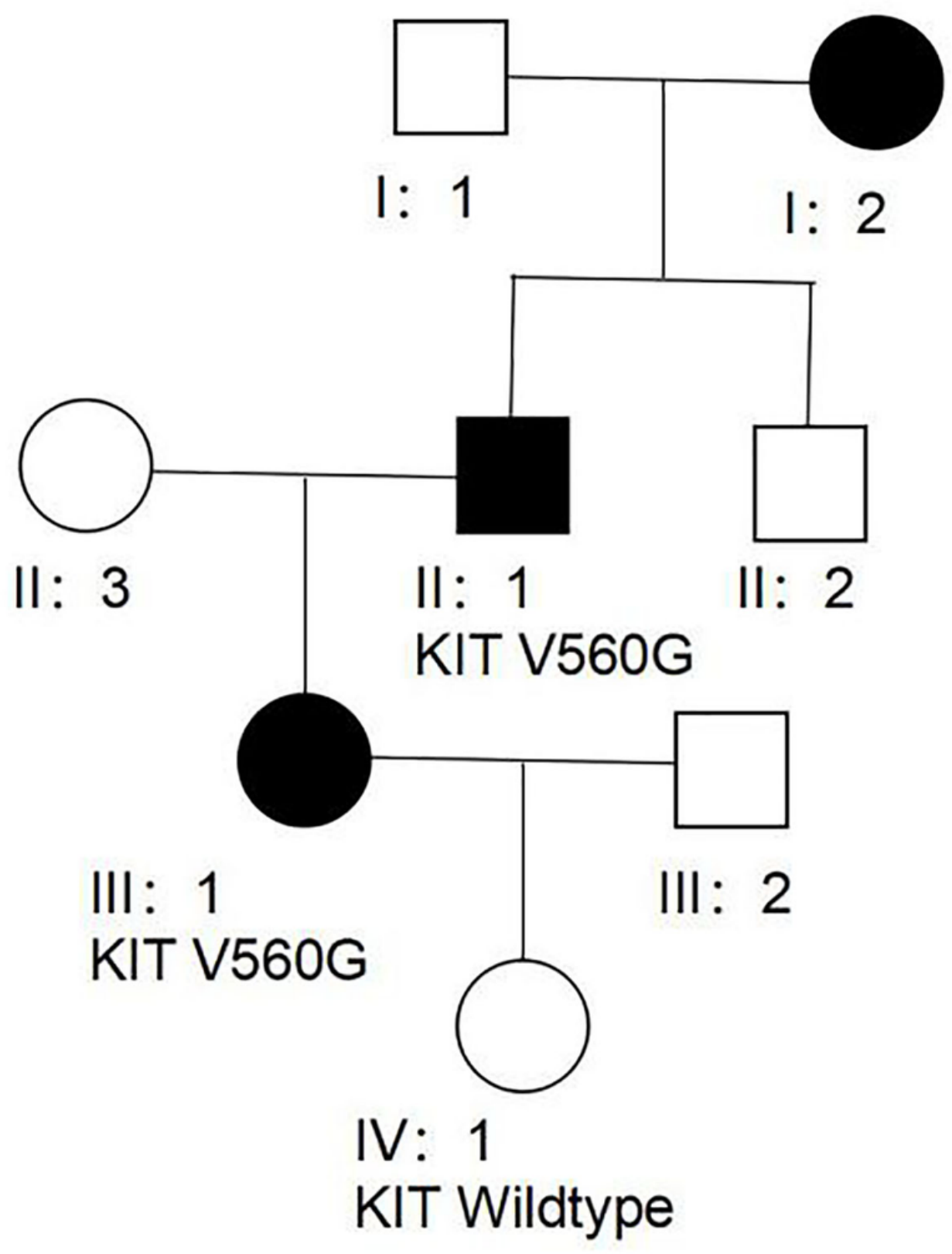

Figure 2

Pedigree of the family. 

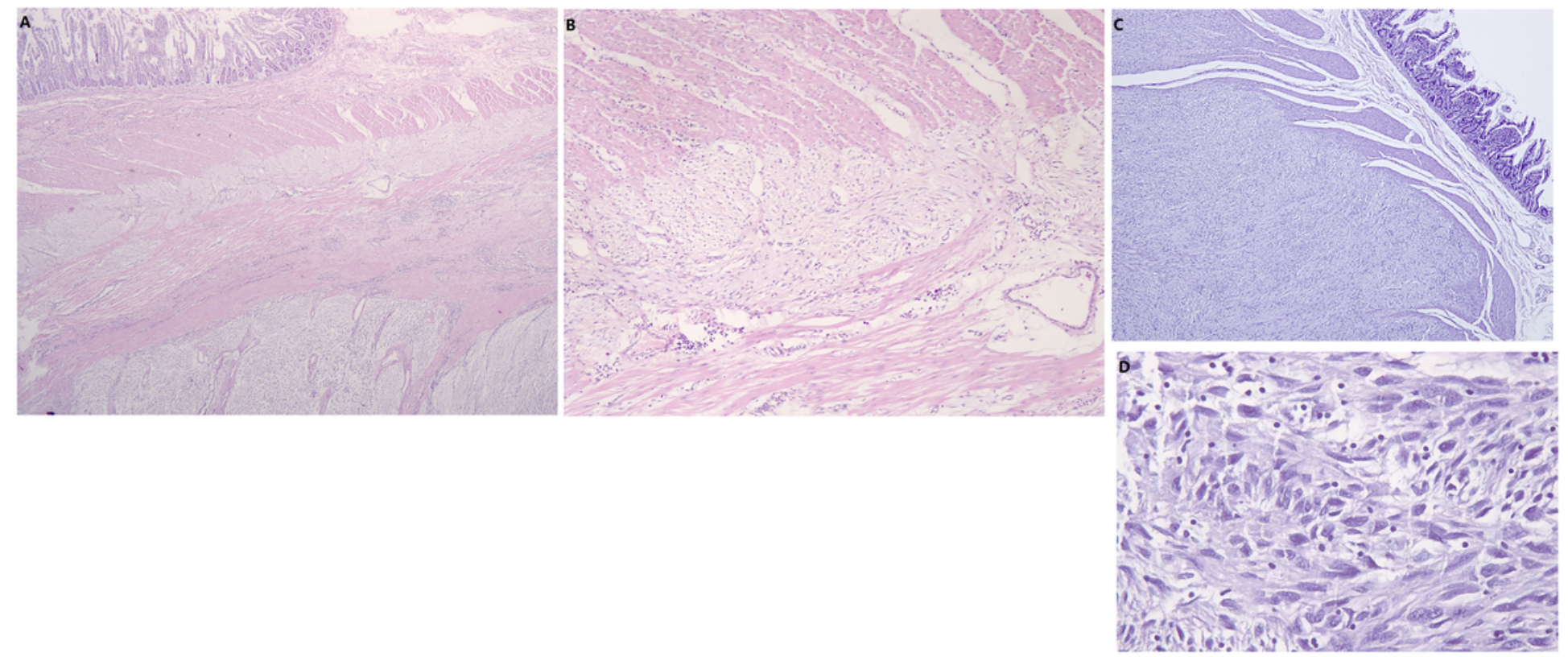

\section{Figure 3}

Morphologic evidences for the role of the interstitial cell of Cajal as the cell of origin of GIST. (A) the transition from the non-neoplastic area to the tumor indicating a hyperplasia of the interstitial cells(original magnification: $\times 2.5$ ). (B) In non-neoplastic area, hyperplasia of interstitial cells of Cajal can be seen (original magnification: $\times 40$ ). (C) (D) The tumor is composed of proliferation of spindle cells (original magnification: $\mathrm{C}: \times 10, \mathrm{D}: \times 20$ ). 